\title{
A mutational hot spot in keratin 10 (KRT 10) in patients with epidermolytic hyperkeratosis
}

Joseph A.Rothnagel1,2, Monte P.Flsher ${ }^{1}$, Shelli M.Axtell', Mark R.Pittelkow ${ }^{3}$, Ingrun Anton-Lamprecht Marcel Huber, Danlel Hohl'5, and Dennis R.Roop ${ }^{1,2, *}$

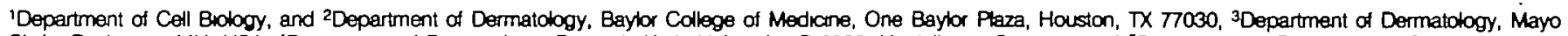
Clunic, Rochester, MN, USA, ${ }^{4}$ Department of Dermatology, Ruprectit-Karls University, D-6900, Hesdelberg, Germany and 5 Depertment of Dermatology, CHUV, Hoprtal

Belmont, Lausanne, Switzerkand

Received July 28, 1993; Revision received September 22, 1993; Accepted October 11, 1993

Epidermolytic hyperkeratosis (EHK), (bullous congenital ichthyosiform erythroderma), Is an autosomal dominant human skin disorder. Recently, we and others have described mutations in keratins 1 and 10 (K1 and K10) in patients with this disease. Structure-function models predict that these mutations would impair normal fllament assembly and functlon. We have extended our earlier studles to include 8 more incidences of EHK. In half of these familles, we were unable to locate a mutation withln the rod domalns of elther K1 or K10. However, polymorphic restriction site and sequence analysis of the other familles revealed a mutatlonal hot spot withln the $1 \mathrm{~A}$ alpha-helical segment of K10. These Involve Arginine to Histidine, Arginine to Cystelne and Arginine to Leucine substitutions at residue 10 of the rod domain. Interestingly, mutations in the corresponding Arginine residue In keratin K14 have been Identified In patlents with epidermolysis bullosa simplex. The large number of mutations found at this position in both keratins K10 and K14 suggests that other eplthella cell disorders will be discovered that are caused by the corresponding mutation In related type I keratin genes.

Epidermolytic Hyperkeratosis (EHK), (bullous congenital ichthyosiform erythroderma), is a genetic human skin disorder transmitted in an autosomal dominant mode with little intrafamilial phenotypic variation (McKusick \#11380 (1)). At birth, affected individuals usually show erythroderma and widespread blistering of the skin. With age, the erythroderma resolves, blistering diminishes and hyperkeratotic lesions become the prominent feature (2). Blistering and erythroderma are mostly absent in adults but can occur as transitory events after physical trauma, stress or treatment with retinoids. The incidence of this disease is estimated at 1 in 200,000 in the United States (3). Histopathology is characterized by cytolysis and intra-epidermal cleavage in the suprabasal layers and a broadening of the granular and stratum corneum layers $(4,5)$. The ultrastructural hallmark of EHK is the appearance of a collapsed keratin filament network with clumps and aggregates of the tonofilaments surrounding the nucleus in the spinous and granular cells of the epidermis $(6,7)$. Ultrastructural studies have long implicated a defect in the differentiation-specific keratins, $K 1$ and $K 10$ as the cause of the disease (6-9). Moreover, several studies have linked the disease to the type II keratin loci on chromosome $12 q(10-12)$. Recently, we and others have described mutations in highly conserved regions of $\mathrm{K} 1$ and $\mathrm{K} 10$ in patients with this disease $(13-15)$. Structure function models, as well as in vitro mutagenesis experiments, predict that these mutations would impair normal filament assembly leading to a collapse of the filament network and clumping of the tonofilaments around the nucleus (reviewed in 13).

Most of the mutations (5 out of 6 ) causing EHK that have been identified to date occur within highly conserved sequence motifs at the beginning and end of the rod domain of these keratins (see Fig. 3). Here, we report four more independent families with EHK that harbor mutations in K10. All of these involved substitutions of the arginine residue at position 10 of the $1 \mathrm{~A}$ domain. In one of the families (EHK-E/S), the disease can be traced back four generations and in the other cases (EHK-A, EHK-K, EHK-D), the mutations were sporadic. The affected individual of the EHK-A family exhibits hyperkeratosis with little or no blistering, even as a child. Most involvement is on the dorsal side of feet and lower extremities with minimal involvement on the backs of hands or neck. There is no involvement of the palms and soles. The face and scalp are clear with normal hair and nails. The affected individual of the EHK-K family suffers from a more generalized expression of the disease. He exhibits hyperkeratosis with blistering and occasional secondary pyroderma over the face, scalp, neck, trunk and extremities as well as hyperkeratosis without blistering of palms and soles. Likewise, the affected individual of the EHK-D family also suffers from massive, generalized hyperkeratoses over the face, scalp, neck, trunk, and extremities, including the palms and soles. All affected members of the EHK-E/S family show generalized involvement of the entire integument, with marked

* To whom correspondence should be addressed 
A EHK - ESS
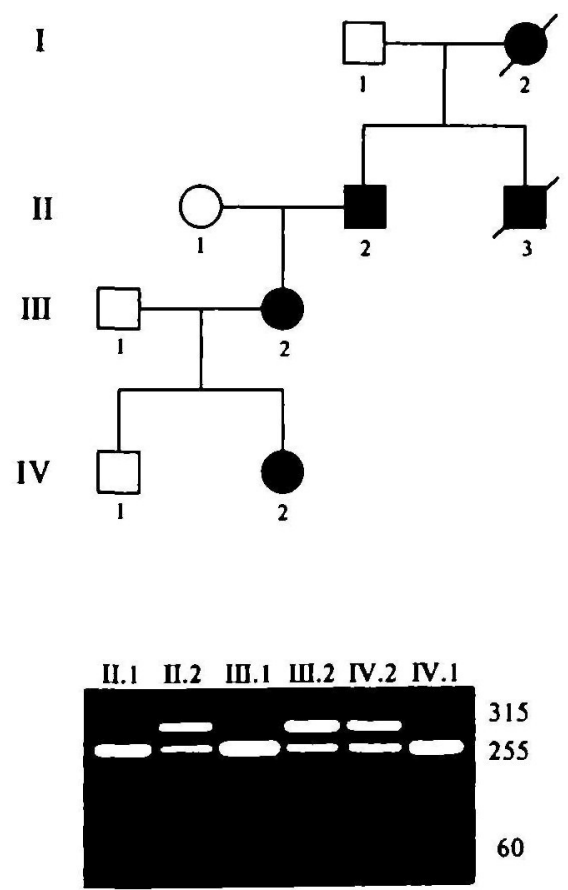

$\mathbf{B}$

Hurmen K10 IA Seqment

Figure 1. (A) Pedigree and PCR analysis of the EHK-E/S family. Males are represented by squares, females by circles and affected individuals by solid symbols. K10 oligonucleotide primers and PCR conditions are as previously described (13). PCR products were digested with Aci I (New England Biolabs, Beverly, MA). (B) Sequence of the beginning of the 1A segment of HK10. The amino acid residues are numbered with respect to the start of the rod domain (13). The arginine at position 10 in this sequence corresponds to residue 156 in K10 (22) and residue 125 in the basal cell type I keratin, K14 (24). The Aci I recognition sequence is boxed and the three substitutions resulting in the loss of this site are indicated.

hyperkeratosis of the scalp, trunk, extremities, backs of hands and feet but sparing the palms and soles. Hair and nails are normal. The individual denoted as I.2 in this pedigree (Figure 1A) appears to have been the spontaneous new mutation, according to information supplied by her spouse and son. A complete clinical description and ultrastructural data from the EHK-E/S proband have been reported elsewhere $(7,16)$.

These mutations were initially identified by Aci I digest of a polymerase chain reaction (PCR) fragment that spans the 1A segment of K10 (Figure 1A, and data not shown). The Aci I recognition sequence includes the arginine codon at position 10 of the rod domain (Figure 1B). The specific mutations were subsequently identified by direct DNA sequencing (Figure 2) using the protocol described previously (13). The PCR product generated by these primers is 315 base pairs (bp) long and after
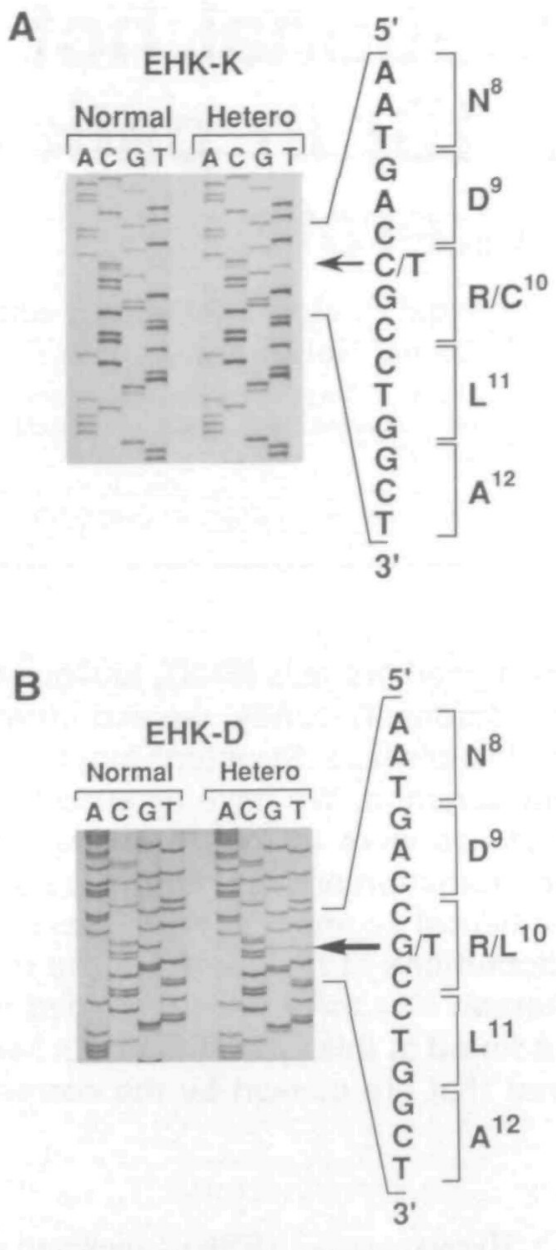

Figure 2. Direct sequencing of PCR products spanning the $1 \mathrm{~A}$ region of $\mathrm{K} 10$ from the EHK-K and EHK-D probands. PCR and sequencing primers are listed in reference 13. Normal, sequence obtained from an unaffected family member. Hetero, sequence from the affected individual. (A) Sequence of the 1A segment of $\mathrm{K} 10$ from the EHK-K proband. The C-T transition is indicated by the arrow and results in the expression of a cysteine residue at position 10 of the rod domain. (B) Sequence of the 1A segment of K10 from the EHK-D proband. The G-T transversion is indicated and results in the expression of a lacine residue at position 10 of the rod domain.

digestion with Aci I, the wild type sequence is cut into a 255 bp and a 60 bp fragment. Patients are heterozygous for the mutant allele and therefore exhibit three fragments in this assay. As can be seen from Figure 1, the Aci I restriction site polymorphism co-segregates with the expression of the disease. Unaffected family members all exhibited the wild type sequence at this site. Moreover, a survey of this site by Cheng et al (14) showed no polymorphisms in a sample of 103 unaffected individuals. Sequence analysis of affected individuals in both the EHK-E/S and EHK-A families revealed a histidine substitution at position 10 of the 1A segment (data not shown). This same substitution has already been reported in three other unrelated incidences of EHK $(13,14)$ and interestingly, in the corresponding position within $\mathrm{K} 14$ in a patient with epidermolysis bullosa simplex (EBS) (17). Keratin K14 is the basal cell homolog of the suprabasal keratin, K10. The EHK-K proband harbors a cysteine substitution at this position (Figure 2A). Although this is the first report of this mutation for EHK, the same substitution has been observed in K14 in a patient with EBS (17). The EHK-D proband shows 


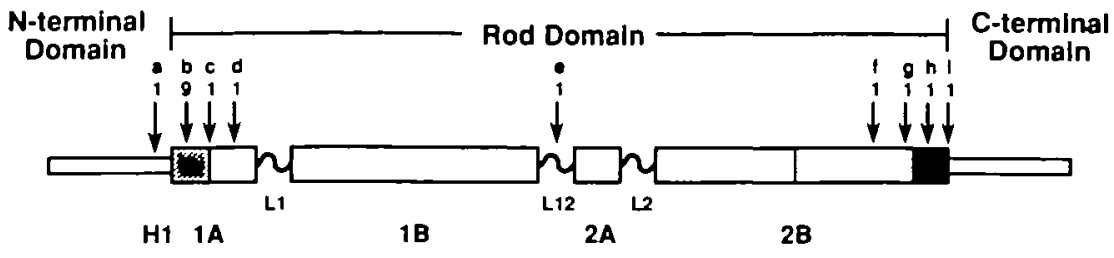

Figure 3. Schermatic showing the positions of mutations reported to date for the keratin skin diseases, EBS and EHK. The conserved sequence motifs at the beginning of the lA segment and at the end of the 2B segment are indicated by hatched and solid boxes, respectively. Data compiled from the present study and: [a] $\mathrm{K} 1$, $\mathrm{L}^{17}$ to $\mathrm{P}$, (H1 subdomain) EHK, Chipev et al 1992 (15); [b] K14, $\mathrm{R}^{10}$ to $\mathrm{C} / \mathrm{H}$, EBS, Coulombe et al 1991 (17); K10, R ${ }^{10}$ to H, (two incidences) EHK, Cheng et al 1992 (14); K10, R10 to H, EHK, Rothnagel et al 1992 (13); [c] K10, L 15 to S, EHK, Rothnagel et al 1992 (13); [d] K14, E29 to A, EBS, Hovnanian et al 1993 (25); [e] K14, M160 to R, EBS, Humphries et al 1993 (26); [f] K14, L272 to P, EBS, Bonifas et al 1991 (27); [g] K5, L295 to P, EBS, Dong et al 1993 (28); [h] K5, E307 to G, EBS, Lane et al 1992 (29); [i] K1, E ${ }^{310}$ to Q, EHK, Rothnagel et al 1992 (13). **Residues are numbered with respect to the H1 subdomain or to the beginning of the rod domain.

an arginine to leucine substitution at this position which is the first report of this substitution in a keratin gene (Figure 2B).

The complete segregation of these arginine mutations with the expression of the disease (this study and refs. 13, 14, 17) coupled with the complete absence of polymorphic variations at this site in unaffected individuals (14) suggests that mutations of this arginine residue are fully penetrant for the disease. It has been observed however, that the first sporadic incidence of EHK in a family may present a less severe clinical picture than their offspring, but never vice-versa, and is attributable to gonadal mosaicism rather than to variable expression of the disease (18). Although it remains to be demonstrated in transgenic studies that the mutations described above are causal, in vitro studies have implicated this arginine residue as critical to filament integrity. The same arginine to histidine substitution in human lamin A was found to destabilize the nuclear lamin filament network (19) and the cysteine mutation in the corresponding position in K14 disrupted the keratin filament network when transfected into cultured keratinocytes (17).

Sequence analysis of the other four families did not reveal any mutations within the rod domains of $\mathrm{K} 1$ and $\mathrm{K} 10$ in affected individuals. Interestingly, Cheng et al., 1992 (14) also failed to find the genetic defect in another four cases of EHK. This suggests that the causative mutations in these patients reside outside the rod domain of $\mathrm{Kl}$ and $\mathrm{K} 10$ or alternatively, that these individuals harbor mutations in other suprabasally expressed keratins, such as K2 (20). Remarkably, three other unrelated incidences of EHK and two of EBS involve substitutions of the arginine residue at position 10 of the rod domain (Figure 3). By comparison, no other position has been associated to date with more than one incidence of mutation. This suggests that this residue is a hot spot for mutations in both $\mathrm{K} 10$ and $\mathrm{K} 14$ and we predict for other type I keratins as well.

A plausible explanation for the apparent sensitivity of this arginine residue to mutation is that its codon contains a $\mathrm{CpG}$ dinucleotide on both coding and non-coding strands. The CPG dinucleotide is acted upon by eukaryotic methylases and subsequent deamination of the methylated cytosine will generate a $\mathrm{C}$ to $\mathrm{T}$ transition (see 14 and refs. therein). Moreover, an increased frequency of transversions have also been observed at these sites (21). CpG dinucleotides also occur at other similarly crucial sites within the rod domain of these keratins such as in the codon for the arginine residue at position 304 of K10, located within the highly conserved TYRXLLEGE motif (22) at the end of the rod domain. In spite of the fact that this region has been extensively analyzed (see Figure 3), not a single mutation has been reported at this residue to date, although in vitro data $(19,23)$ have shown that it is critical for filament function. This suggests that other factors, such as the degree of methylation, may be involved in determining the mutational hot spot observed at the arginine residue $\left(\mathrm{R}^{10}\right)$ within the rod domain of type I keratins.

\section{ACKNOWLEDGEMENTS}

We thank Jackie Bickenbach and Janelle Laminack for help with preparation of this manuscript. We also thank the patients and their families for their cooperation. This work was supported by a NIH grant to DRR (HD25479) and a grant from the Swiss National Science Foundation to DH (31-30323-90).

\section{REFERENCES}

1. McKusick, V.A. (1992) Mendelian Inheritance in Man, 10th (ed) Johns Hopkins University Press, Baltimore.

2. Brocq, L. (1902) Ann. Dermatol. Syph. 4, 1-31.

3. F.I.R.S.T. registry (1992)

4. Frost, P. and Van Scott, E. (1966) Arch. Dermatol. 94, 113-126.

5. Ackerman, A.B. (1970) Arch. Dermatol. 102, 253-259.

6. Weibel, E. and Schnyder, U. (1966) Arch. Klin. Exp. Dermatol. 255, 286-298.

7. Anton-Lamprecht, I. and Schnyder, U.W. (1974) Arch. Derm. Res. 250, 207-227.

8. Anton-Lamprecht, I. (1983) J. Invest. Dermatol. 81, 149s-156s.

9. Ishida-Yamamoto, A., McGrath, J.A., Judge, M.R., Leigh, I.M., Lane, E.B. and Eady, R.A.J. (1992) J. Invest. Dermatol. 99, 19-26.

10. Bonifas, J.M., Bare, J.W., Chen, M.A., Lee, M.K., Slater, C.A., Goldsmith, L.A. and Epstein, E.H. (1992) J. Invest. Dermatol. 99, 524-527.

11. Compton, J.G., DiGiovanna, J.J., Santraci, S.K., Keams, K.S., Amos, C.I., Abangan, D.L., Korge, B.P., McBride, O.W., Steinert, P.M. and Bale, S.J. (1992) Nature Genet. 1, 301-305.

12. Pulkkinen, L., Christiano, A.M., Knowiton, R.G. and Uitto, J. (1993) J. Clin. Invest. 91, 357-361.

13. Rothragel, J.A., Dominey, A.M., Dempsey, L.D., Longley, M.A., Greenhalgh, D.A., Gagne, T.A., Huber, M., Frenk, E., Hohl, D. and Roop, D.R. (1992) Science 257, 1128-1130.

14. Cheng, J., Syder, A.J., Yu, Q.-C., Letai, A., Paller, A.S. and Fuchs, E. (1992) Cell 70, 811-820.

15. Chipev, C.C., Korge, B.P., Markova, N., Bale, S.J., DiGiovanna, J.J., Compton, J.G. and Steinert, P.M. (1992) Cell 70, 821-828.

16. Anton-Lamprecht, I. (1977) Z. Hautker. 52, 809-811.

17. Coulombe, P.A., Hutton, M.E., Letai, A., Hebert, A., Paller, A.S. and Fuchs, E. (1991) Cell 66, 1301-1311.

18. Traupe, H. (1989) The Ichythoses. Springer-Verlag.

19. Heald, R. and McKeon, F. (1990) Cell 61, 579-589.

20. Collin, C., Moll, R., Kubicka, S., Ouhayoun, J.-P., and Franke, W.W. (1992) Exp. Cell Res. 202, 132-141.

21. Bottema, C.D.K., Bonema, M.J., Keuterling, R.P., Voon, H.-S., Janco, R.L., Phillips, J.A., and Sommer, S.S. (1991) Am. J. Hum. Genet. 49, 839-850. 
2150 Human Molecular Genetics, 1993, Vol. 2, No. 12

22. Rieger, M. and Franke, W.W. (1988) J. Mol. Biol. 204, 841-856.

23. Letai, A., Coulombe, P., McCornick, M.B., Yu, Q.-C., Hutton, E., and Fuchs, E. (1993) Proc. Natl. Acad. Sci. USA 90, 3197-3201.

24. Marchuk, D., McCrohon, S., and Fuchs, E. (1984) Cell 39, 491-498.

25. Hovnanian, A., Pollack, E., Hilal, L., Rochat, A., Prost, C., Barrandon, Y. and Gossens, M. (1993) Nature Genetics 3, 327-332.

26. Humphries, M.M., Sheils, D.M., Farrar, G.J., Kumar-Singh, R., Kenna, P.F., Mansergh, F.C., Jordon, S.A., Young, M. and Humphries, P. (1993) Human Mutation 2, 37-42.

27. Bonifas, J.M., Rothman, A.L. and Epstein, E.H. (1991) Science 254, $1202-1205$.

28. Dong, W., Ryynanen, M. and Uito, J. (1993) Human Mutation 2, 94-102.

29. Lane, E.B., Rugg, E.L., Navsaria, H., Leigh, I.M., Heagerty, A.H.M., Ishida-Yamamoto, A. and Eady, R.A.J. (1992) Nature, 356, 244-246. 